\title{
Undergraduate student involvement in global health
}

\author{
Anji E. Wall* \\ Vanderbilt University Medical Center, Nashville, USA
}

Over the past several years, there has been a surge of interest in global heath in the medical community. This seems particularly prevalent in pre-medical undergraduate students, as they generally have the luxury of time to participate in global health activities. I had the opportunity to speak about global health at an undergraduate institution a few months ago, to a room full of over 100 pre-medical students. All were interested in global health and about $50 \%$ had participated in a global health mission. During my visit, the pre-med advisor commented that global health experience is becoming another check box on the medical school application. The question that has been bothering me since then is: should medical schools encourage undergraduate students with no formal medical education or experience to participate in global health missions?

This is a very personal question as I participated in a medical mission in Guatemala as a junior in college and have been grappling with a feeling of ambivalence to the point of discomfort ever since. Briefly, I and 10 other undergrads spent 3 weeks in Guatemala on a service based mission that involved a reforestation project and pouring concrete for the roof of a library among other things. A group of medical students was also in the area at the same time, and because of my interest in medicine, I was invited to tag along with them as they set up mobile clinics in some of the rural areas. These clinics were set up in a different place each day, usually in a public building or school. Hundreds of patients were seen, diagnosed (most commonly with infectious diseases), and provided medications free of charge. When medications were not available, the group provided vitamins to patients for nutritional supplementation. This was an eye opening experience in that it vividly showed me the burden of disease in a developing country and the true lack of access to health care that many individuals are plagued with.

The questions that still bother me are: Did we do any good? Did we help anyone? Should I, as someone without any knowledge of medicine, have been seeing patients and passing out medications? We gave out a lot of medications that were seemingly appropriate but we did not have any laboratory tests to corroborate diagnoses nor did we have any way to follow up with patients to make sure that their conditions improved and that they were not having serious side effects. Moreover, we did nothing to improve their future access to health care or to address the underlying causes of their medical conditions (e. g., providing access to safe water to decrease the incidence of amoebic dysentery). When I look back at my overall experience in Guatemala, I am most proud of the library roof that I helped build, and not of the rural clinics that I attended. I hope that when I finish my long road of residency training, I will be poised to return to the developing world with the skills necessary to provide interventions or surgical education that will improve health and health care. But, ten years after my trip to Guatemala, I feel less certain of my ability to provide meaningful interventions in global health, probably because every day of residency provides new challenges and uncertainty in a setting that has excellent oversight and seemingly unlimited resources.

To return to the issue at hand, in many respects, undergraduate students are ideal candidates for assisting in global health missions. They have an abundance of time off, especially during the summer months. They have a lot of energy. They are itching for hands on medical experience to get a sense of if this is truly the career that they want to be a part of. They are also driven to get into medical school and if this sort of experience provides an advantage (or levels the playing field) then they are going to pursue it. Moreover, early introduction to global health missions has been shown to increase volunteer activity both at home and abroad.

However, undergraduate pre-medical students are not ideal candidates in other respects. The most glaring issue is that most undergraduate students have no formal training in health care. In the United States, these students are often given the opportunity to observe physicians but not to participate in the care of patients. That sort of restriction is not generally the case in medical missions to developing countries. I have heard accounts of pre-medical students delivering babies and repairing lacerations. While this is an amazing story for them to tell, and patients generally do okay after these interventions, setting a double standard with respect to patient care is ethically questionable. In addition, premedical students can be faced with situations in which they are not prepared to provide necessary medical care. Vulnerable patients in developing countries may not be informed or understand the lack of training that these students have and may expect them to function as independent providers even though they are not poised to do so. This can set up uncomfortable and even frightening situations. If a patient dies or has a bad outcome in the hands of a premedical student, this can cause serious remorse and regret, possibly pushing them away from pursing a medical career. More importantly, the poor outcome will not only affect the patient and the patient's family, but also the community's perception of medical volunteers.

There are so many initiatives that undergraduates can participate in that will improve the health and well-being of communities in developing countries without having them participate directly in medical care. For example, they can assist with public health initiatives such as providing mosquito nets for malaria prevention or gathering survey data that helps define the current state of health care in areas of the developing world so as to identify targeted interventions that

Correspondence to: Anji E. Wall, $\mathrm{MD}, \mathrm{PhD}$, Vanderbilt University Medical Center, 116121 st Ave S, CCC-4312 MCN, Nashville, TN37232-2730, USA, Tel: 615-343-6642; Fax: 615-322-0689; E-mail: neilshethmd@gmail.com

Received: October 24, 2015; Accepted: November 18, 2015; Published: November 21, 2015 
address the needs of communities. These types of initiatives introduce undergraduate students to global health in a way that they can provide needed interventions, while at the same time, protecting them from participating in hands-on health care that they are not trained to provide.

Don't get me wrong. Improving health and health care in developing countries is an imperative and getting students interested and involved at an early stage in their development will likely contribute to greater understanding of the global burden of disease and build a generation of physicians who want to be part of the solution. However, we need to encourage participation in initiatives that allow premedical students to take an active role without requiring medical training so as to protect them from situations in which they are not able to provide the care necessary for sick patients and to protect patients from the provision of healthcare by untrained individuals. As surgery takes a more prominent role in global health, it is imperative that the surgical community takes a leading role in determining if and how pre-medical students are involved in these endeavors.

Copyright: @2015 Wall AE. This is an open-access article distributed under the terms of the Creative Commons Attribution License, which permits unrestricted use, distribution, and reproduction in any medium, provided the original author and source are credited. 